\title{
Endogenous Control and Task Representation: An fMRI Study in Algebraic Problem-solving
}

\author{
Andrea Stocco and John R. Anderson
}

\begin{abstract}
The roles of prefrontal and anterior cingulate cortices have been widely studied, yet little is known on how they interact to enable complex cognitive abilities. We investigated this issue in a complex yet well-defined symbolic paradigm: algebraic problem solving. In our experimental problems, the demands for retrieving arithmetic facts and maintaining intermediate problem representations were manipulated separately. An analysis of functional brain images acquired while participants were solving the problems confirmed that prefrontal regions were affected by the retrieval of arithmetic facts, but only scarcely by the need to manipulate intermediate forms of the equations, hinting at a specific role in memory retrieval. Hemodynamic activity in the dorsal cingulate, on the contrary, increased monotonically as
\end{abstract}

\section{INTRODUCTION}

Humans are set apart from other species by their ability to perform sequences of arbitrary operations over complex representations. Such activities include planning, problem solving, and the wide range of symbol-manipulation abilities that probably underlie language and certainly enable mathematical and logical reasoning. These capabilities are usually credited to an increased capacity for cognitive control, namely, the ability to voluntarily coordinate the ongoing cognitive processes in order to achieve a certain goal.

A large part of the literature on this topic has been concerned with basic experimental paradigms, such as the Stroop or the Flanker task, or task switching (e.g., Yeung, Nystrom, Aronson, \& Cohen, 2006; Sohn, Ursu, Anderson, Stenger, \& Carter, 2000; Botvinick, Nystrom, Fissell, Carter, \& Cohen, 1999; Pardo, Pardo, Janer, \& Raichle, 1990). But even under such controlled conditions, it is often difficult to distinguish control functions from the processing of task-relevant information. In task switching, for instance, the so-called switch cost is usually assumed to reflect the additional control effort for reconfiguring the response set. However, the same effect has been also interpreted as a cost paid in retriev-

Carnegie Mellon University, Pittsburgh, PA more information processing steps had to be taken, independent of their nature. This pattern was essentially mimicked in the caudate nucleus, suggesting a related functional role in the control of cognitive actions. We also implemented a computational model within the Adaptive Control of Thought-Rational (ACT-R) cognitive architecture, which was able to reproduce both the behavioral data and the time course of the hemodynamic activity in a number of relevant regions of interest. Therefore, imaging results and computer simulation provide evidence that symbolic cognition can be explained by the functional interaction of medial structures supporting control and serial execution, and prefrontal cortices engaged in the on-line retrieval of specific relevant information.

ing appropriate information from memory (Logan \& Bundesen, 2003; Altmann \& Gray, 2002), a lack of benefit from successive task repetitions (Sohn \& Anderson, 2001), and as the interference between the alternated task representations (Yeung et al., 2006). Also, the contribution of different cognitive strategies to accomplishing the same task might be confounded with executive control. As an example, Lovett (2005) presented a model of the Stroop task that was capable of reproducing most of the effects reported in the literature as the result of an internal competition between alternative procedures. As another example, the strategy-conflict model of Peebles and Bothell (2004) succeeded in reproducing participant's performance in the sustained-attention-toresponse task.

By using both computational modeling and brain imaging, our research group has previously succeeded in tracking the maintenance of temporary representations and the adoption of cognitive strategies in more complex domains (Anderson, Albert, \& Fincham, 2005; Fincham, Carter, Van Veen, Stenger, \& Anderson, 2002), and the effects of practice and learning (Fincham \& Anderson, 2006; Qin et al., 2003). In this article, the same approach is used to examine the interplay between task-related and control-related information in algebraic problem solving, a domain which had been investigated in a series of previous imaging studies (e.g., Anderson, 2005; Qin et al., 2003, 2004). 


\section{The Role of the Anterior Cingulate Cortex and the Lateral Prefrontal Cortex in Cognitive Control}

Two brain regions have been mainly characterized as underlying cognitive control: the lateral part of prefrontal cortex (LPFC) and anterior cingulate cortex (ACC). Although the contribution of LPFC in cognitive control and executive functions is commonly agreed upon (e.g., Miller \& Cohen, 2001), the consensus is less on its exact functional role. Braver, Cohen, and Barch (2002) pointed out that there are at least two issues at stake: whether PFC is seen as a passive memory buffer or an active controller, and, in the latter case, whether its functions are inhibitive or attentive. The less controversial finding probably is its contribution to memory, especially working memory (e.g., Wagner, Paré-Blagoev, Clark, \& Poldrack, 2001; Nolde, Johnson, \& D'Esposito, 1998).

The anterior part of the cingulate gyrus is a complex structure involved in several functions. Its most dorsal part (dACC), however, has been repeatedly found to correlate with internal control demands (e.g., Bush, Luu, \& Posner, 2000). Three major views have been put forward: According to the earliest theory, the dACC is responsible for the orienting of attention, and particularly for recruiting the brain regions required for the execution of a particular task (Posner \& Dehaene, 1994; Pardo et al., 1990). A subsequent hypothesis was that ACC was monitoring behavior and detecting errors, providing fundamental feedback for correcting one's own actions (Dehaene, Posner, \& Tucker, 1994). A third view has been recently gaining prominence, according to which the dACC detects conflicts among competing responses (Carter et al., 2000; Botvinick et al., 1999). Botvinick, Cohen, and Carter (2004) suggested a two-step framework, where the dACC detects conflict and, before a selection among competing responses is made, it alerts PFC to adjust the amount of top-down control. The balancing of control between the two regions has been empirically supported (MacDonald, Cohen, Stenger, \& Carter, 2000) and can also explain the same data supporting the error detection hypothesis (Yeung, Botvinick, \& Cohen, 2004).

The conflict-monitoring hypothesis is noteworthy because it fractionates cognitive control into two subsystems, one for detecting conflict and one for resolving it through top-down selection, making one important step toward a more mechanistic explanation of control. However, it must be noted that ACC has been found to be sensitive to a wide range of control demands, not limited to conflict monitoring (e.g., Badre \& Wagner, 2004). Additionally, lesion studies in humans have brought forth inconsistent results: Some authors (Di Pellegrino, Ciaramelli, \& Làdavas, 2007; Swick \& Turken, 2002) found evidence of reduced ability in adapting to control demands in patients with focal damage to ACC, whereas others (Baird et al., 2006; Fellows \& Farah, 2005) did not.

\section{Cognitive Control within a Cognitive Architecture}

Our experiment and our predictions were based on the Adaptive Control of Thought-Rational (ACT-R) cognitive architecture (Anderson et al., 2004). In ACT-R, representations are temporarily held in independent information processing modules, corresponding to different cortical regions. Communication between modules is made possible by a central, procedural module, which detects the onset of particular conditions through the incoming pathways from the cortex and routes the relevant information to the appropriate regions. The action of relaying information among modules corresponds to the minimal cognitive steps, and is computationally encoded as production rules. The activity of this central module has been related to functions of the basal ganglia (Anderson, 2005; Anderson et al., 2004). Although the basal ganglia are involved in several cognitive and noncognitive functions (e.g., Graybiel, 2000), this interpretation is consistent both with their role in procedural knowledge acquisition (Knowlton, Mangels, \& Squire, 1996) and with existing computational models stressing their role as a gating system to PFC (O'Reilly \& Frank, 2006).

Being a production system, ACT-R implicitly assumes a definition of control that does not correspond to the hierarchical models we have reviewed. From a purely computational perspective, control is achieved by the serial execution of production rules-this being all that determines the transitions of the system from one state to another. Instead of a hierarchy of controllers, a production system achieves flexible behavior by building upon progressively more articulated representations, therefore banishing the need for a control homunculus. Correspondingly, dichotomies such as top-down versus bottom-up control, or controlled versus automatic behavior, scarcely make any sense because representations lay on a continuum of complexity.

The role of appropriate representations in the control of action has been increasingly appreciated in recent years. For instance, Botvinick and Plaut (2004) presented a neural network whose capability for coordinating routine sequences of actions depends on the number of layers and how remote they are from (and, therefore, less interfered with by) immediate sensory units. When units were removed, the model produced patterns of errors that closely mimicked those found in frontal patients. Similarly, Koechlin, Ody, and Kouneiher (2003) provided evidence that, moving from the caudal to the rostral part of the frontal lobe, prefrontal regions encode increasingly complex information (from simple sensory input to contextual signals to episodic memory). Also, Schneider and Logan (2005) presented a task switching model that can fully account for the various types of switch cost by relying only on memory processes involved in the retrieval of appropriate response representations. 


\section{Specialized Control Information and the Role of the Anterior Cingulate Cortex}

Previous studies have been able to identify distinctive brain regions whose hemodynamic activity could be related to different ACT-R modules (Anderson, 2005; Anderson et al., 2004). In particular, one part of the LPFC located within the inferior frontal gyrus was found to correlate with the demands for memory retrievals. These demands correspond to the activity of a retrieval module in ACT-R. Also, a posterior parietal region has been mapped onto ACT-R's imaginal module that holds and updates problem-state information, and a region in the motor cortex to the manual module that programs manual output. As previously noted, the procedural module has been associated with the basal ganglia and, in particular, with the activity of the caudate nucleus.

One ACT-R component is of special interest for our purposes: it is the so-called goal module, which has been mapped onto the dACC (Fincham \& Anderson, 2006; Anderson, 2005). Its functional role is to hold controlrelevant information that relates to the ongoing process. Special control information is necessary to make a selection whenever more than one basic behavioral action is compatible with the current state of the modules. This happens in situations of response conflict but will also occur when there are no responses being selected. Control states do not need to have semantic content, and their role is often limited to providing introspective access to the internal states of cognitive processing modules. This view has the advantage of reducing the gap between the cognitive functions of ACC and its involvement in monitoring and regulating somatic responses (e.g., Critchley et al., 2003).

In any case, the need for control information increases in complex tasks where more representations need to be processed and maintained in different modules. This very same factor also affects the procedural module because more representations are usually needed when larger amounts of information are being relayed. In a cognitive-demanding task such as algebra, the hemodynamic activity in the dACC and in the striatum should be similarly affected by manipulating the internal demands for maintaining and retrieving information.

\section{THE EXPERIMENT}

Cognitive control has been traditionally studied with the paradigms we outlined previously, which often contrast trials where an automatic response is appropriate with trials where voluntary actions are needed to inhibit them. Here, we tried to keep both task and stimuli constant, but to manipulate the amount control that is necessarily performed internally ("endogenous") versus the amount of control that is explicitly encoded in the stimuli themselves ("exogenous": Monsell, 2003). The task we opted for was solving simple algebraic equations. Algebraic problem solving is complex and arbitrary enough to significantly engage control and specialized task representations, yet well enough understood to allow for mechanistic modeling. In addition, brain regions involved in representing different types of algebraic knowledge have been previously identified (Anderson, 2005; Qin et al., 2003).

\section{Stimuli and Design}

Our participants were presented with 128 equations, ${ }^{1}$ all of which comprised of four terms, two on the left and two on the right of the equal sign ("="). Each equation was either entirely parametric or entirely numeric. An example of parametric equation is reported in Equation 1:

$$
a * x-a=a * b-a
$$

whereas Equation 2 gives one of the numeric type:

$$
8 * x-2=36-6
$$

The leftmost term was always the only one containing the unknown quantity $x$. It always contained another factor, which either multiplied (e.g., " $8 * x$," " $a * x$ ") or divided (e.g., " $x / 4, "$ " $x / \mathrm{b}$ ") the unknown.

The solution of each equation required three steps: (a) Eliminating the addend on the left side by appropriately adding or subtracting a quantity; (b) Unwinding the unknown by applying the inverse operand of its factor; and eventually, (c) Providing the correct result. Participants were required to perform these steps in this exact order. At each step, the correct action was selected by pressing the key corresponding to one out of four possible options. In the first two steps, the options were the four possible operators ("+," “-," "**," and " $/$," respectively). In the last step, the options were either " 2 ," "4," "6," and " 8 " (for numeric equations) or $a, b$, $-a$, and $-b$ (for the parametric ones), which were also the only possible results of each equation. For instance, in solving Equation 2, participants needed to select " + " (to get rid of the left addend " -2 ") and then "/" (to unwind the unknown in " $8 * x$ ") from the list of operators, and eventually pick up the correct answer ("4") among the possible results (see Figure 1).

Four conditions were obtained by manipulating two variables within subjects: (a) whether equations contained parameters or numbers, and (b) whether the equation was updated internally or externally. In the external condition, the software calculated and displayed the intermediate version of the equation resulting from the application of the selected option after each response 
Figure 1. Design of our experiment. The two series of panel represent the time courses of two possible trial types, with either internal (top panels, in gray) or external update (bottom panels, in black). Equations could be numeric or parametric (not depicted), and were always followed by a white panel whose duration was stretched to give each trial a fixed duration of $22.5 \mathrm{sec}$. All the problems required exactly three behavioral responses in the form of right-hand finger presses, corresponding to the three steps needed to solve each equation (eliminating the left addends, eliminating the factor in the main term, and giving the result, respectively).

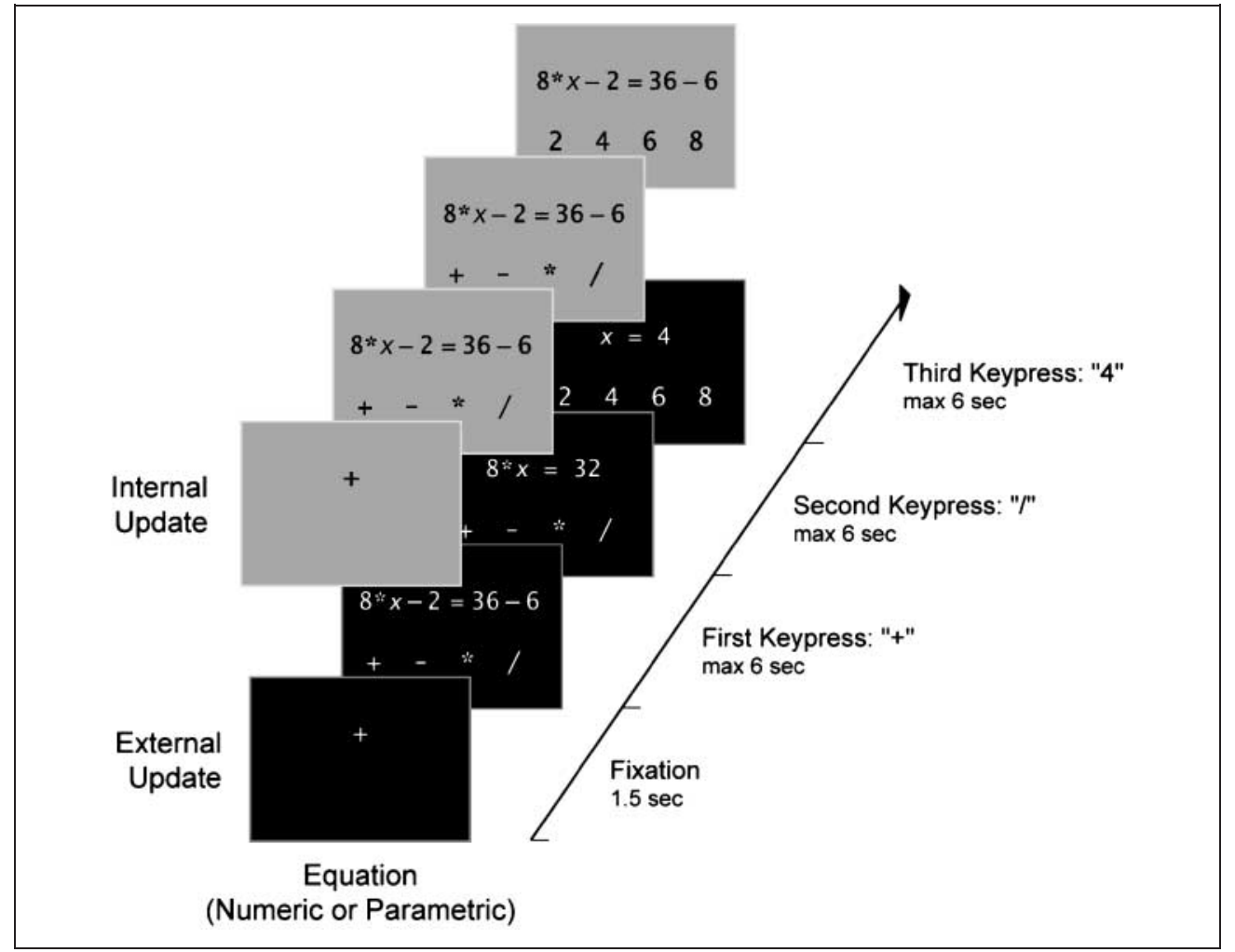

(see Figure 1). Conversely, when the update was internal, the software kept displaying the initial version of the equation, forcing participants to represent the intermediate forms within their heads. All the problems were displayed in black characters. The background color was light blue when the update was external and yellow when it was internal (see Figure 1). Problems were randomly mixed and separated by variable-length blank screens with a white background.

\section{Predictions}

A large network of prefrontal and parietal areas is engaged by the execution of complex tasks (Cole \& Schneider, 2007; Hill \& Schneider, 2006). However, the increasing representational demands of our experimental conditions should affect differently certain regions. Our view is that the control of behavior is mainly due to the activity of medial frontal and subcortical structures (and, in particular, the basal ganglia and ACC), whereas prefrontal and parietal cortices are mostly engaged in maintaining specific types of task information. The current task relies on two kinds of representations: (a) arithmetic knowledge retrieved during numeric calculations; and (b) intermediate states of the problem that must be maintained and updated during the solution process, and which we assumed to be represented as imagery of how the equations would be written after each operation. Our experimental conditions pose different demands on these two types of representations. The two external conditions do not require any of them because everything is taken cared of by the software. Internal parametric problems, on the other hand, require storing and manipulating an inner representation of the equation in order to solve it. Finally, internal numeric problems require both the manipulation of intermediate equation forms and the retrieval of arithmetic knowledge.

Our expectations were as follows. We predicted that there should be no difference between the two types of equations when the update was external. Given our identification of the LPFC with ACT-R's retrieval buffer, we expected it to be mainly sensitive to retrieval demands only, and therefore, its hemodynamic response to be higher for the internal numeric problems than for the others. Therefore, our prediction for this prefrontal region was: internal numeric $>$ internal parametric $=$ external (numeric $=$ parametric). In contrast, activity in both ACC and the caudate nucleus should exhibit a monotonic increase in activation from the simplest to the most difficult condition. The reason for this is that both retrieving arithmetic knowledge and maintaining intermediate problem states increase the amount of control states required and the amount of information transferred during cognitive operations. Therefore, our predictions for both cingulate and caudate nucleus were: internal numeric $>$ internal parametric $>$ external (numeric $=$ parametric). 


\section{METHODS}

\section{Participants and Task}

Participants were 15 normal, right-handed college students (age $=18-24$ years, $M=20.3$ years, $S D=1.7$, 7 women). Shortly before the beginning of the experimental session, they were given written instructions about the task and a short computer-based practice session consisting of eight equations. An additional practice of 16 equations was given at the beginning of the scanning session, while high-resolution structural images were acquired. ${ }^{2}$ None of the practice equations were later used in the experiment. During the experiment, participants were presented with 128 different problems (32 per condition) grouped into 8 consecutive blocks of 16 trials each, with a short pause between each block. The equations were presented in random order.

Participants' responses were recorded through a data glove attached to their right hand. Stimuli presentation and response collection were conducted using the EPrime (Psychological Software Tools, Pittsburgh, PA) software package.

\section{Image Acquisition and Analysis}

Imaging data were collected with a Siemens 3-T Allegra Scanner using a standard radio-frequency head coil. Each functional volume contained 29 oblique axial slices (3.2 $\mathrm{mm}$ thickness, FOV $=200 \mathrm{~mm}, 64$ by 64 matrix, 3.125 by $3.125 \mathrm{~mm}$ in-plane resolution) parallel to the AC-PC plane, with the 20-sec slice from the superior centered at the AC-PC line. Functional images were acquired using a gradient echo-planar image (EPI) acquisition sequence $(\mathrm{TR}=1500 \mathrm{msec}, \mathrm{TE}=30 \mathrm{msec}$, flip angle $=73^{\circ}, \mathrm{FOV}=200 \mathrm{~mm}$, zero slice gap). Functional acquisition was event-related, with image acquisition synchronized to stimulus onset, such that 15 volumes, each with 29 slices, were acquired during each 22.5-sec trial. There were eight functional imaging blocks, each containing 16 experimental trials.

Anatomical images were acquired using a standard T1weighted spin-echo pulse sequence at the identical slice location as the functional images using a finer in-plane resolution $(3.2 \mathrm{~mm}$ thickness, FOV $=200 \mathrm{~mm}, 256$ by 256 matrix, 0.78 by $0.78 \mathrm{~mm}$ in-plane resolution).

Preprocessing of the functional imaging data included six-parameter rigid-body motion correction using AIR (Woods, Cherry, \& Mazziotta, 1992). Data were spatially transformed into a common space using the transformation gotten from coregistering anatomical images to a common reference structural MRI image ${ }^{3}$ by means of a 12-parameter automatic algorithm AIR (Woods, Grafton, Holmes, Cherry, \& Mazziotta, 1998), and then smoothed with a 6-mm full-width half-maximum 3-D Gaussian filter to accommodate individual differences in anatomy.

\section{BEHAVIORAL RESULTS}

Data from one participant were discarded because his mean latencies were more than three standard deviations above the average in all conditions. The mean reaction time for each of the three responses was calculated for each condition and averaged over all participants. Only correct trials were included in the analysis. Figure 2 plots the average reaction times for the three responses in a trial, with the individual line plots representing the four conditions. Table 1 contains the comprehensive latencies (from the equation onset to the final answer) in the correct trials, as well as the average accuracy across all problems, for each condition.

Performance in the external numeric and external parametric problems was very similar relative to the large differences among the other conditions. They differed in terms of latency $[t(13)=2.82, p=.01]$ but they did not on accuracies $[t(13)=1.16, p=.26]$. As expected, the
Figure 2. Participants mean latencies by response and condition (solid lines), together with the correspondent predictions by our model (dotted lines).

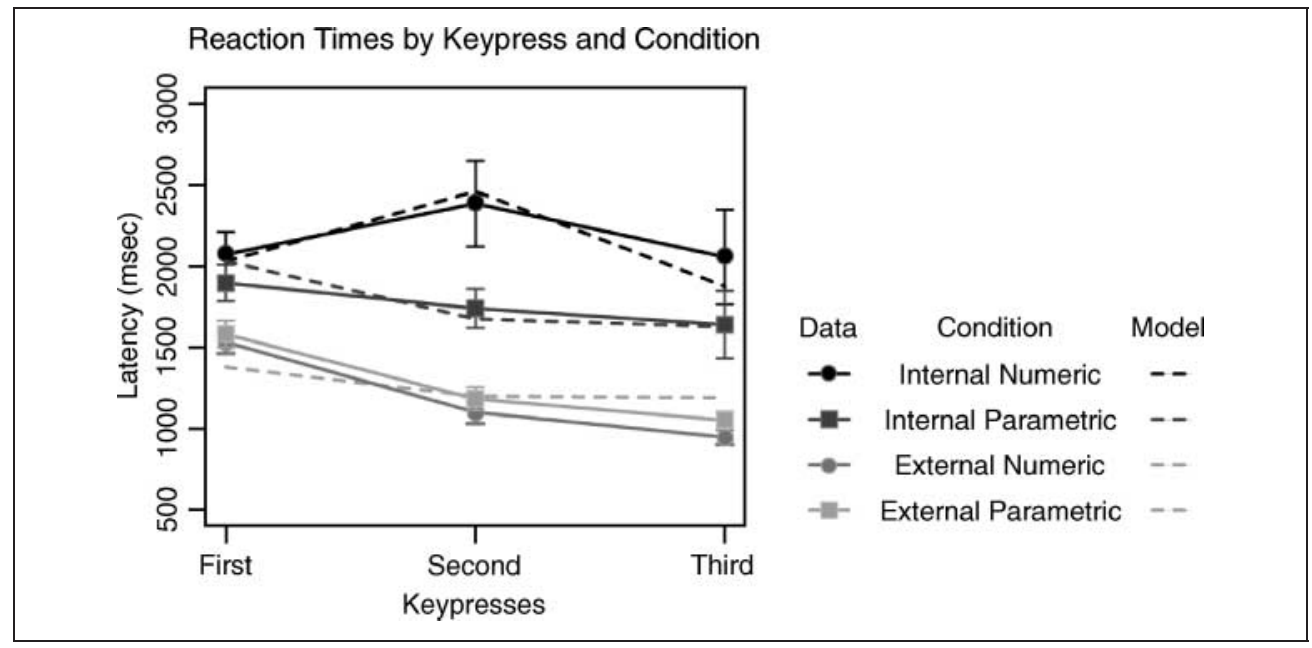


Table 1. Behavioral Results

\begin{tabular}{lcc}
\hline \multirow{2}{*}{ Update } & \multicolumn{2}{c}{ Equation } \\
\cline { 2 - 3 } Internal & Numeric & Parametric \\
& $6514(242)$ & $5277(216)$ \\
External & $0.75(0.03)$ & $0.87(0.03)$ \\
& $3579(125)$ & $3820(162)$ \\
& $0.96(0.01)$ & $0.95(0.01)$ \\
\hline
\end{tabular}

Mean latency (standard error) $[\mathrm{msec}]$ and, in the line below, mean accuracy (standard error) by equation and update type.

additional representations that had to be processed in the other conditions exerted significant tolls. The internal parametric condition was more difficult than its external counterpart, as indexed by decreased accuracy $[t(13)=-2.36, p=.03]$ and higher latencies $[t(13)=$ $10.42, p<.0001]$. Similarly, the internal numeric condition was significantly more difficult than the internal parametric [reduced accuracy, $t(13)=-2.89, p=.01$; and higher latency: $t(13)=4.97, p=.0003$ ].

\section{CONFIRMATORY ANALYSIS}

Five predefined regions of interest (ROIs) were used in the confirmatory analysis, all of them located in the left hemisphere. These regions constitute a subset of larger group of 16 that had been previously mapped onto corresponding modules of the ACT-R architecture (e.g., Anderson, 2005, 2007; Anderson et al., 2004). They are marked in black in Figure 3 and listed in Table 2, together with their corresponding anatomical structure, their size, their computational counterpart, and the TalairachTournoux coordinates of their centroids.

It must be noted that, despite our design being apparently factorial, the retrieval of arithmetic facts was not supposed to take place in the external but only in the internal numeric problems. The two external conditions (parametric vs. numeric) were not predicted to differ, and, as expected, in none of the predefined regions was the hemodynamic activity significantly dissimilar between them. Therefore, instead of analyzing our data as 2 by 2 factor manipulation, we excluded the external numeric problems and concentrated on the three remaining conditions. These conditions vary monotonically in terms of behavioral difficulty and representational demands: The internal parametric condition differs from the external parametric because it requires the maintenance of intermediate equation forms, whereas the internal numeric differs from its parametric counterpart because it additionally requires the retrieval of arithmetic knowledge.

All the incorrect and the first trials of each block were excluded from the analysis. The blood oxygenation leveldependent (BOLD) response for each trial was computed
Figure 3. Seven of the eight regions identified in our exploratory analysis (in red; ROI 8 is below the displayed slices) and the five predefined ROIs (in black) overlaid onto 18 axial slices of our reference brain. The AC-PC line passed through Slice 22 (not shown).

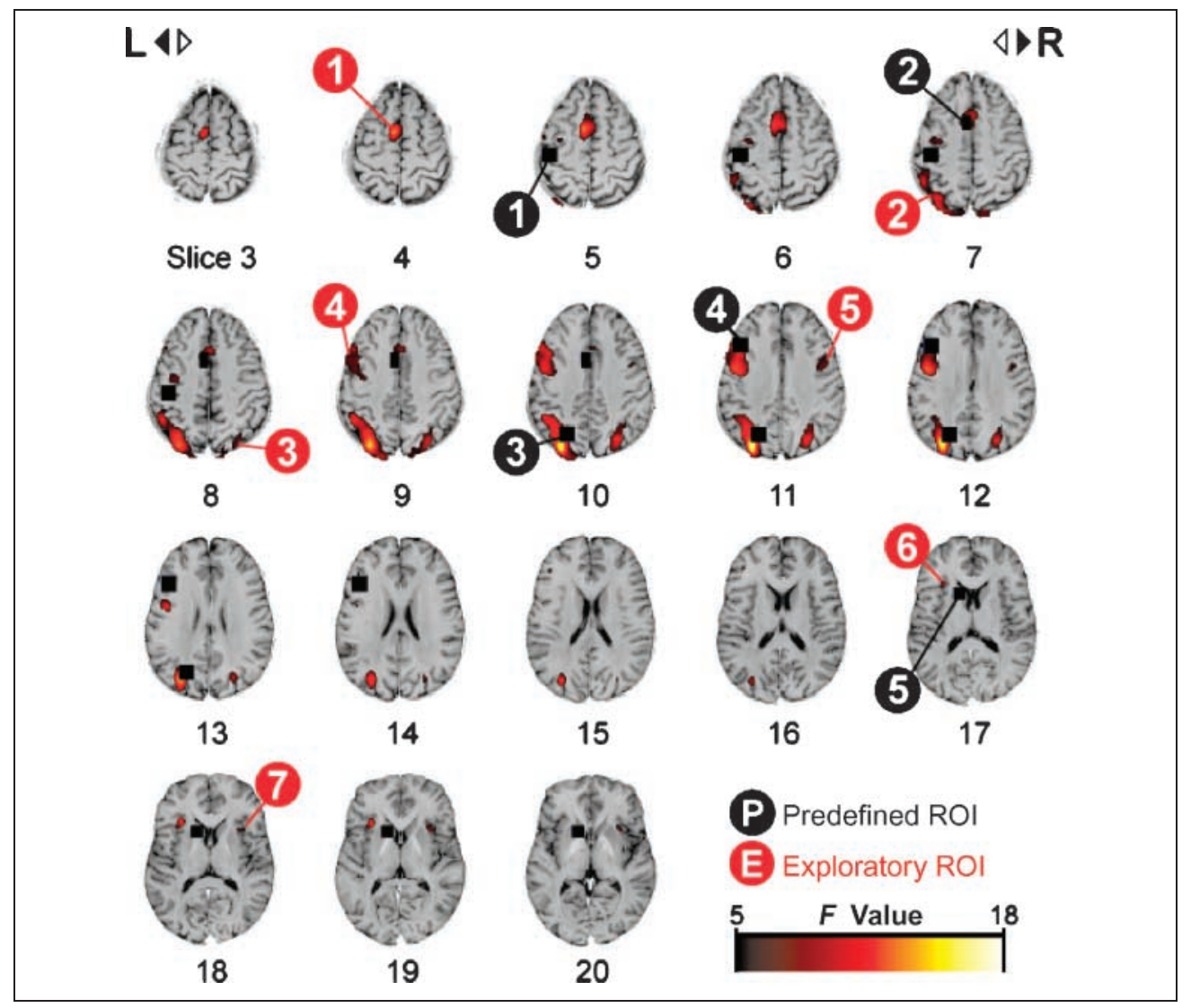


Table 2. Summary of Confirmatory Analysis

\begin{tabular}{|c|c|c|c|c|c|c|c|}
\hline \multirow[b]{2}{*}{$R O I$} & \multirow[b]{2}{*}{$\begin{array}{c}\text { Gray Matter } \\
\text { (Brodmann's Area) }\end{array}$} & \multirow[b]{2}{*}{$\begin{array}{l}\text { Corresponding } \\
\text { ACT-R Module }\end{array}$} & \multirow[b]{2}{*}{$\begin{array}{c}\text { Size } \\
\text { (Voxels) }\end{array}$} & \multirow[b]{2}{*}{$\begin{array}{c}\text { Talairach-Tournoux } \\
\text { Coordinates }\end{array}$} & \multicolumn{3}{|c|}{ Areas under the Curve } \\
\hline & & & & & $\begin{array}{c}\text { External } \\
\text { Parametric }\end{array}$ & $\begin{array}{c}\text { Internal } \\
\text { Parametric } \\
(t \text { Value, vs. } \\
\text { External })\end{array}$ & $\begin{array}{c}\text { Internal } \\
\text { Numeric } \\
\text { (t Value, vs. } \\
\text { Parametric) }\end{array}$ \\
\hline 1 & $\begin{array}{l}\text { Precentral gyrus } \\
\quad \text { (BA 3) }\end{array}$ & Manual & $5 \times 5 \times 4$ & $-42,-20,50$ & 4.85 & $4.55(-0.66)$ & $4.88(0.51)$ \\
\hline 2 & $\begin{array}{l}\text { Anterior cingulate } \\
\text { cortex (BA 24/32) }\end{array}$ & Goal & $3 \times 5 \times 4$ & $-6,10,39$ & 4.09 & $5.11(2.27 *)$ & $6.94(2.54 *)$ \\
\hline 3 & $\begin{array}{l}\text { Posterior parietal } \\
\text { lobule, precuneus } \\
\text { (BA 7/39) }\end{array}$ & Imaginal & $5 \times 5 \times 4$ & $-24,-63,40$ & 5.27 & $6.82\left(3.15^{* *}\right)$ & $7.98(2.27 *)$ \\
\hline 4 & $\begin{array}{l}\text { Middle frontal gyrus } \\
\text { (BA 9/46) }\end{array}$ & Retrieval & $5 \times 5 \times 4$ & $-42,23,24$ & 2.69 & $2.60(-0.35)$ & $5.32(5.51 * *)$ \\
\hline 5 & $\begin{array}{l}\text { Head of the caudate } \\
\text { nucleus }\end{array}$ & Procedural & $4 \times 4 \times 4$ & $-13,10,7$ & 1.99 & $2.66(2.86 *)$ & $4.02(3.61 * *)$ \\
\hline
\end{tabular}

The five predefined ROIs (and their correspondent ACT-R modules) used in the confirmatory analysis. The last three columns contain the average positive areas under the curve for each condition, together with the $t$ values obtained by contrasting the two parametric (internal vs. external update) and the two internal (numeric vs. parametric) conditions.

$* p<.05$, uncorrected, $d f=13$.

$* * p<.01$, uncorrected, $d f=13$.

using the last data scan from the previous trial as the baseline from which percent change was calculated over the full time course of the trial. For each subject and for each ROI, trials belonging to the same condition were averaged together. As a measure of the overall activity elicited by different conditions in each brain region, the positive part of the area under the hemodynamic curve was also calculated for each subject. We adopted this measure because it is not compromised by pre- and postpeak negative dips that are frequently present in the BOLD response, and it is sensitive to both the absolute magnitude of the response and its duration (Anderson, 2005). The area under the curve was then used to calculate two critical contrasts for each region: (a) between the two parametric conditions (internal vs. external update), to examine its sensitivity to the update factor; and (b) between the two internal conditions (numeric vs. parametric), to examine its sensitivity to the retrieval factor. The results of these contrasts are listed in the last two columns of Table 2, while Figure 4 displays the time course of the BOLD response (averaged across subjects) in the predefined regions.

The first of our predefined regions corresponds to the part of the left motor cortex that is responsible for hand movements, and has been mapped to ACT-R's manual module. The varying difficulty across conditions should have no impact on its overall activity (because the amount of motor programming remains equal) but should be reflected in a significant Scan by Condition interaction because of the increased lag between finger presses (see
Figure 2). As expected, both our contrasts turned out to be nonsignificant $[t(13)>0.66, p>.52]$. A repeated measures analysis of variance (ANOVA) was run on the averaged BOLD responses, using scan (16 values) and condition (3 values) as within-subject factors. In this and all the subsequent analysis, subject was always treated as a random factor. The results conformed to our predictions [Effect of condition: $F(2,26)=0.72, p=.50$; interaction: $F(30,390)=3.08, p<.0001$; see Figure 4, Plot 1].

Three other ROIs exhibited the pattern we predicted for control-related regions, with the hemodynamic response increasing monotonically across condition, and both contrasts being significant. These regions are located in the dACC, in the posterior parietal cortex, and in the head of the caudate nucleus (see Table 3 and Figure 3 , regions marked in black). The contrast between the internal numeric and the external parametric conditions was also significant $[t(13)>3.78, p<.003$, uncorrected], as well as the main effect of condition on the areas under the curve $[F(2,26)>9.68, p<.001]$.

The BOLD response in our predefined ACC and caudate regions conformed to our predictions (see Figure 4, Plots 2 and 5). A comparison between the BOLD responses in ACC and the caudate nucleus yielded a nonsignificant ROI by Condition interaction $[F(2,26)=1.83$, $p=.18]$, suggesting that they were similarly impacted by the increased demands for control.

The predefined left prefrontal region also conformed to our prediction, showing no significant difference between the two parametric conditions, but a significantly larger re- 
Figure 4. Time course of the BOLD response for the three critical conditions in our five predefined ROIs (solid lines), together with the corresponding hemodynamic responses predicted by the model (dotted lines). In each plot, the first scan is the baseline, whereas the following 15 belong to the trial.
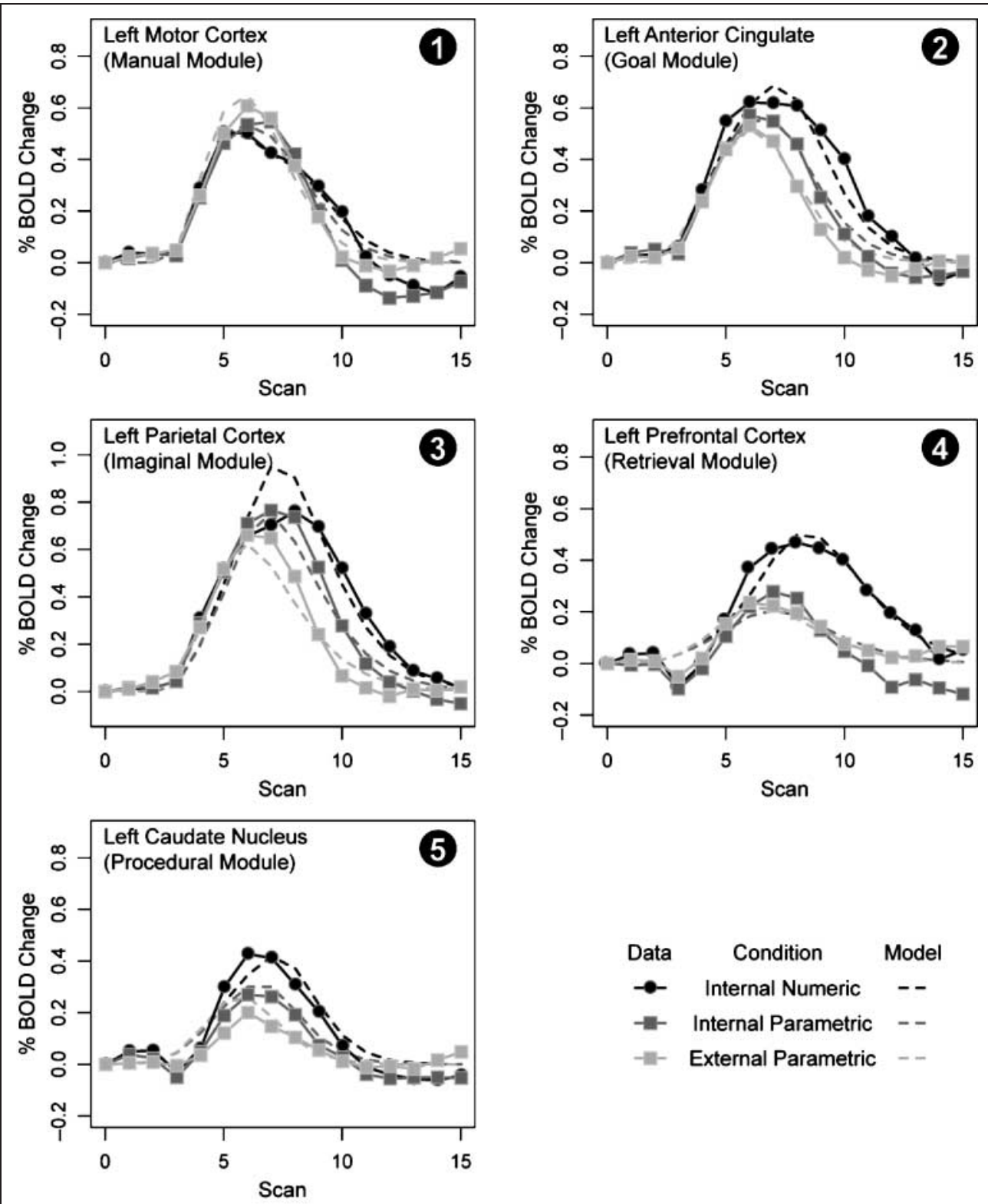

sponse for the internal numeric problems (see Table 3). This pattern is consistent with the increased demands for retrievals elicited in the internal numeric condition.

The difference between our lateral prefrontal and anterior cingulate regions was further tested by means of a Scan (16 values) by Condition ( 3 values) by ROI ( 2 values) ANOVA. A significant main effect of ROI $[F(1,13)=$ $5.36, p=.04]$ and a significant interaction between ROI and condition $[F(2,26)=3.41, p<.05]$ were found, confirming that different problem types had a different effect on those brain areas. The three-way interaction between scan, condition, and ROI was also significant $[F(30,390)=1.89, p=.004]$. The same interaction held when comparing the hemodynamic response in our predefined prefrontal region with the head of the caudate nucleus [Condition by ROI interaction: $F(2,26)=7.04$, $p=.004$; Condition by ROI by Scan: $F(30,390)=6.95$, $p<.0001$ and the left posterior parietal cortex [Condition by ROI interaction: $F(2,26)=5.44, p=.01$; Condition by ROI by Scan, $F(30,390)=4.29, p<.0001]$.

\section{Discussion}

Our confirmatory analysis essentially supports our predictions. We found responses of identical magnitude in the motor region and the characteristic sensitivity of the lateral PFC to arithmetic retrievals. Consistently with their involvement in cognitive control, monotonically larger responses were found in the AACC and the caudate nucleus.

\section{EXPLORATORY ANALYSIS}

An exploratory analysis was used to identify other regions reliably responding to the manipulations of the 
Table 3. Summary of Exploratory Analysis

\begin{tabular}{|c|c|c|c|c|c|c|c|}
\hline \multirow[b]{2}{*}{$R O I$} & \multirow[b]{2}{*}{$\begin{array}{c}\text { Gray Matter } \\
\text { (Brodmann's Area) }\end{array}$} & \multirow[b]{2}{*}{$\begin{array}{c}\text { Size } \\
\text { in Voxels }\end{array}$} & \multirow[b]{2}{*}{$\begin{array}{l}\text { Talairach- } \\
\text { Tournaoux } \\
\text { Coordinates }\end{array}$} & \multirow[b]{2}{*}{$\begin{array}{l}\text { Mean (Max) } \\
\text { F Value }\end{array}$} & \multicolumn{3}{|c|}{ Areas under the Curve } \\
\hline & & & & & $\begin{array}{c}\text { External } \\
\text { Parametric }\end{array}$ & $\begin{array}{l}\text { Internal } \\
\text { Parametric } \\
(t \text { Value, vs. } \\
\text { External })\end{array}$ & $\begin{array}{l}\text { Internal } \\
\text { Numeric } \\
\text { ( } t \text { Value, vs. } \\
\text { Parametric) }\end{array}$ \\
\hline 1 & Left anterior cingulate (BA 32) & 240 & $-2,14,43$ & $7.05(14.14)$ & 4.26 & $5.63(3.36 * *)$ & $8.27(3.49 * *)$ \\
\hline 2 & $\begin{array}{l}\text { Left superior parietal lobule } \\
\text { (BA 7) }\end{array}$ & 678 & $-29,-58,48$ & 7.78 (17.98) & 7.05 & $8.32\left(2.15^{*}\right)$ & $10.51\left(4.08^{* *}\right)$ \\
\hline 3 & $\begin{array}{l}\text { Right superior parietal lobule } \\
\text { (BA 7) }\end{array}$ & 237 & $27,-62,45$ & $6.33(10.08)$ & 7.35 & $8.46(2.06)$ & $10.56(2.89 *)$ \\
\hline 4 & $\begin{array}{l}\text { Left middle frontal gyrus } \\
\text { (BA 9) }\end{array}$ & 395 & $-40,12,31$ & $6.65(12.32)$ & 4.24 & $4.36(0.26)$ & $7.82(5.71 * *)$ \\
\hline 5 & $\begin{array}{l}\text { Right inferior frontal gyrus } \\
\text { (BA 9) }\end{array}$ & 46 & $48,6,32$ & $5.57(6.80)$ & 5.37 & $5.64(0.41)$ & $8.75(5.05 * *)$ \\
\hline 6 & Left insula & 29 & $-28,24,6$ & $7.14(10.41)$ & 2.74 & $3.08(0.52)$ & $5.63(4.41 * *)$ \\
\hline 7 & Right insula & 26 & $35,15,8$ & $5.78(6.90)$ & 2.71 & $3.61(1.98)$ & $5.90\left(2.45^{*}\right)$ \\
\hline 8 & $\begin{array}{l}\text { Left inferior temporal gyrus, } \\
\text { middle occipital gyrus } \\
\text { (BA 19) }\end{array}$ & 64 & $-46,-58,-4$ & $5.80(7.25)$ & 5.09 & $6.67(4.63 * *)$ & $8.13(3.25 * *)$ \\
\hline
\end{tabular}

List of brain regions showing a significant $(p<.01)$ Scan $\times$ Condition interaction when comparing the three critical conditions $($ external parametric vs. internal parametric vs. internal numeric). The last three columns contain the average positive areas under the curves for each condition, together with the $t$ values obtained by contrasting the two parametric (internal vs. external update) and the two internal (numeric vs. parametric) conditions.

$* p<.05$, uncorrected, $d f=13$.

$* * p<.01$, uncorrected, $d f=13$.

experiment. All the incorrect and the first trials of each block were excluded from the analysis. Functional imaging data were analyzed using mixed effects ANOVA models (Braver et al., 1997) within the NIS system (http:// kraepelin.wpic.pitt.edu/nis/). In the analysis, subject was treated as a random factor and within-subject factors included condition and scan (16 values). This analysis was used to identify a number of brain areas that showed differential BOLD profiles in response to different conditions, indicated by a significant Condition by Scan interaction. The lower bound degree of freedom correction was applied to correct for nonsphericity due to nonindependence of scans, using an alpha level of $p<.01$. To correct for multiple comparisons issues, only those regions having a contiguous cluster size of 20 or more significant voxels are reported (Forman et al., 1995).

A preliminary comparison between the two externally updated conditions (numeric and parametric) showed no reliable difference in our fMRI data. Therefore, we concentrated on three critical conditions: external and internal parametric and internal numeric. Here, eight different ROIs matched our criteria: They are marked in red in Figure 3 and listed in Table 3, together with their corresponding anatomical structure, their size, and the Talairach-Tournoux coordinates of their centroids.

For each of these regions, the average BOLD responses and the corresponding area under the curves were calcu- lated for each condition, using the same method as in the confirmatory analysis. The areas under the curves were used to calculate the same two contrasts (external vs. internal parametric and internal parametric vs. numeric). These eight regions divide into two categories. Four of them (ROI 4, 5, 6, and 7) show the same pattern that we predicted for the LPFC: a similar response for the two parametric conditions (whether the update was internal or external), but a significantly larger response for the internal numeric problems. The other four (ROI 1, 2, 3, and 8) exhibit a substantially monotonic pattern, showing higher peaks and larger areas under the curves as difficulty increased.

In the first group, two regions (ROI 4 and 5) are prefrontal clusters comprehending large portions of the left and right inferior and middle frontal gyri (BA9/46; the left one also extends to the precentral gyrus). The left region partly overlaps with our predefined prefrontal ROI (see Figure 3), and both their hemodynamic responses fully conform to our expectations for memory retrievals. The same pattern was also uncovered in the left and right insular region (ROI 6 and 7)

In three of the remaining four regions, both contrasts were significant at $p<.05$ (see Table 2), suggesting that these regions were sensitive to both factors. They consisted of a medial region in the prefrontal lobe, encompassing the supplementary motor area and the dACC 
(ROI 1); a large parietal cluster (ROI 2), centered around the left superior parietal lobule; and a region between the left middle occipital and the left inferior temporal gyri. The first two regions partly overlapped with two of our predefined ROIs (see Figure 3).

In the fourth region (ROI 3), centered in right superior parietal lobule, the contrast between the two external conditions was only marginally significant $[t(13)=$ 2.06, $p=.06$ ]. This region was compared with its left counterpart (ROI 2) to see whether there was any significant difference. A Condition (3 values) by Region (right vs. left parietal) ANOVA on the areas under the curve uncovered no significant main effect of the region $[F(1$, $13)=2.31, p=.15]$ and no significant interaction $[F(2$, 26) $=0.52, p=.60]$. Given the substantial similarity of their hemodynamic responses, it was concluded that our right parietal particle exhibited the same monotonic pattern as its left counterpart.

Two distinct peaks of activity were found in our left parietal cluster: one in the left intraparietal sulcus (Talairach coordinates: $x=-46, y=-44, z=47$ ) and one centered in the left superior parietal lobule (Talairach coordinates: $x=-30, y=-72, z=42$; see Figure 3 ). The first peak was located in close proximity to left horizontal portion of the intraparietal sulcus. This region is the most important of the three circuits identified by Dehaene, Piazza, Pinel, and Cohen (2003), supposedly responsible for a modality-independent representation of numerical magnitude. The second spot was in close proximity of the left precuneus, which is responsive to both mental imagery and episodic memory retrievals (e.g., Fletcher et al., 1995).

The pattern of activation we found in our parietal ROI could actually be the sum of two distinct hemodynamic responses, one due to number processing and one due to mental imagery. To rule out this possibility, two subregions were identified and analyzed separately. The first region was a box of $3 \times 2 \times 2$ voxels $\left(375 \mathrm{~mm}^{3}\right)$ centered on the peak of the left horizontal portion of the intraparietal sulcus, and the second one was a box of $4 \times 4 \times$ 4 voxels $\left(2000 \mathrm{~mm}^{3}\right)$ centered on the precuneus. Their hemodynamic patterns turned out to be remarkably similar. No significant ROI by Condition interaction was found $[F(2,26)=1.02, p=.38]$, suggesting that these two subregions were not responding significantly differently to the same conditions. Similarly, a Condition by ROI ANOVA on the areas under the curves failed to uncover a main effect of the ROI $[F(1,13)=1.48, p=.25]$ and found just a marginally significant interaction $[F(2,26)=$ 3.01, $p=.07]$.

\section{Discussion}

The results of the exploratory analysis are consistent with the confirmatory analysis and conform to our expectations. First, in contrasting the two types of externally updated problems, no regions were reliably affected by the mere presence of numbers versus parameters. Second, when comparing the three critical conditions, the activity in both the left and right prefrontal clusters conformed to our expectations for memory retrieval, being scarcely, if at all, affected by the extra effort in maintaining intermediate problem states, but strongly engaged when arithmetic retrievals were required. This pattern also seemed to be mirrored in the two insular regions.

Also, our medial prefrontal cluster, which crucially encompasses part of our predefined cingulate ROI, conformed to our prediction of larger responses for more demanding problems, and its hemodynamic activity differed significantly from PFC. A very similar hemodynamic pattern was also exhibited by two parietal regions and one occipito-temporal region.

\section{COMPUTATIONAL MODEL OF THE TASK}

Although the strategy to solve an equation is well defined in general terms, a number of degrees of freedom remain-for example, in how to interleave arithmetic retrievals and problem manipulations. More detailed strategies were derived from a comparative analysis of the latency data.

The pattern in Figure 2 provides some informative clues. Reaction times follow almost identical patterns in the two external conditions, implying an identical strategy. Reaction times for the first response are similar in the two internal conditions, and in both cases, larger than in the external problems: this suggests that participants are performing the same series of cognitive operations, which, in turn, rule out the retrieval of arithmetic facts because it cannot occur in the parametric condition. The model assumes that participants use this first step not only to determine whether the response is an addition or a subtraction, but also to encode the right-hand side of the equation. It follows that, in the internal numeric problems, all the arithmetic retrievals must occur in the last two steps. In particular, it was hypothesized that the last two retrievals occur between the second and the third responses, and only the first one (always an addition or subtraction) takes place between the first and the second, compensating for the larger number of mental manipulations occurring in this phase.

Given such constraints, three solution strategies were derived for the four types of equations and implemented in an ACT-R model. ${ }^{4} \mathrm{~A}$ visual rendition of these strategies is depicted in Figure 5. ${ }^{5}$ The figure details the activity of three modules (retrieval, imaginal, and manual, whose contents are more clearly interpretable) while solving exemplificative equations of the three relevant types. In the figure, times flows down the chart, and individual module's periods of activity are represented by boxes on each module's track. The boxes' contents illustrate the 


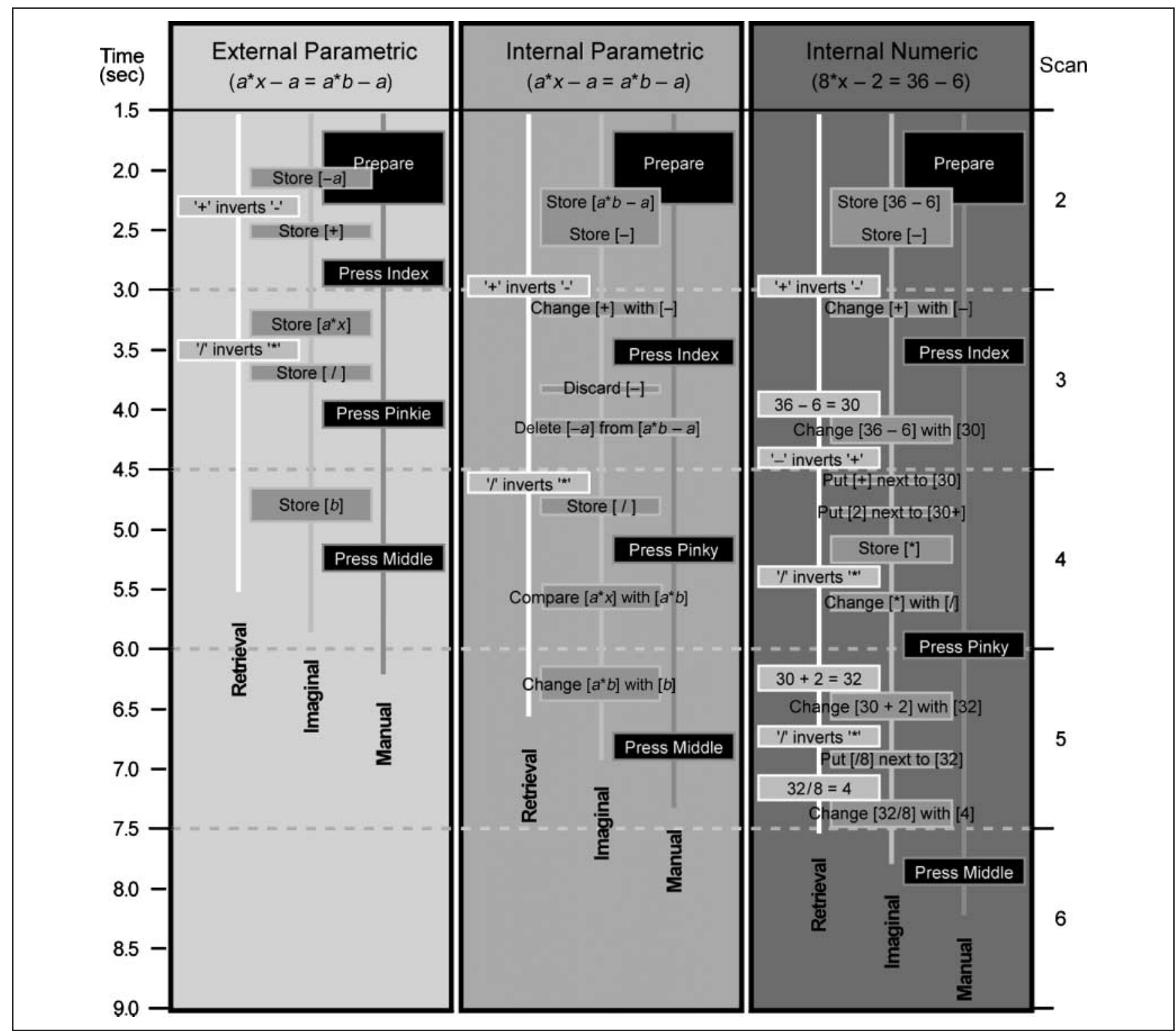

Figure 5. A visual rendition of the strategies implemented by the model, together with their time course. Boxes represent the periods of time during which the three modules (retrieval, in light gray; imaginal, in dark gray; and manual, in black) were active while solving an example trial of each condition. The contents of the boxes illustrate the operations being performed by each module during each period of activity. The timeline starts at the onset of the equation: The fixation period (corresponding to the first scan) is omitted.

operation being performed, whereas their vertical extent reflects the time needed to complete it.

The model replicates our behavioral results $(r=.959$, RMSE $=116.65 \mathrm{msec}$; see the dotted lines in Figure 2). Two parameters were estimated: the first was the latency factor, which scales retrieval times from declarative memory (Anderson et al., 2004), and was set to 0.17. The second parameter was the time needed to complete each manipulation of the contents of the imaginal module and was set at $50 \mathrm{msec}$. This parameter contributed to account for the higher reaction times in the internal numeric condition, where the need to integrate the results of arithmetic calculations within the internal prob- lem representation eventually required a larger number of manipulations in the imaginal buffer (see Figure 5).

Once the model was fit to the behavioral data, the predicted hemodynamic responses were calculated. The BOLD response $B(t)$ for a point event occurring at time $t=0$ was approximated as a gamma function (Glover, 1999):

$$
B(t)=t^{a} e^{-t}
$$

The cumulative BOLD response $\mathrm{CB}(t)$ for a predefined region was calculated by convolving Equation 3 with the 
corresponding module's demand function, as proposed by Anderson (2005) and Anderson, Qin, Sohn, Stenger, and Carter (2003):

$$
\mathrm{CB}(t)=M \int_{0}^{t} D(\tau) B\left(\frac{t-\tau}{s}\right) d \tau
$$

where $D(\tau)$ is the value of the demand function at time $\tau$. Demand functions were calculated for each module by averaging the time course of its activity over all the trials belonging to the same condition. As in previous simulations (e.g., Anderson, 2005), the retrieval module was considered active between the initiation and the completion of a retrieval; the motor module was considered active from the initial preparation of a motor movement through its final realization; and the imaginal module was considered active from the beginning to the end of an internal problem manipulation. The goal module was considered active during the execution of any production rule that modified or tested for a control state. Differently from what has been previously done, activity in the procedural module was calculated as the amount of information relayed by each production being executed. Production rules transfer information by binding values to variables in their condition side, and relaying them to their action side. Therefore, the amount of information transferred was quantified as the number of variable bindings occurring within each production rule.

Both the parameters $a$ in (Equation 3 ) and $s$ in (Equation 4) concur in determining the shape of the response function and its peak onset, whereas $M$ in (Equation 4) scales its amplitude. These parameters were estimated for each module, but remained identical across conditions. Although fit independently, their values were consistently similar across modules (see Table 4).

Figure 4 reports the estimated BOLD responses obtained for each module (dotted lines) together with the original hemodynamic data from our predefined ROIs (solid lines). The last two columns of Table 4 report the correlation coefficients and the root mean squared errors (RMSE) between the predicted and observed responses. The model's predictions correlated significantly with the actual data ( $r>.93$ for all ROIs) and were consistent with the different patterns observed in different regions.

\section{GENERAL DISCUSSION}

This article examined the effect of manipulating the amount of control required to solve algebraic equations by varying the amount and type of the internal representations needed. The lateral prefrontal regions were significantly affected only by increased demands for arithmetic retrievals, and not by other representational loads, even when the latter had significant and large effects on behavior and in other brain regions. This result is in agreement with previous studies where lateral prefrontal activity was dissociated from sheer difficulty (Barch et al., 1997), and its contribution to control was identified with holding on-line information (Cole \& Schneider, 2007). On the contrary, the dACC and the caudate nucleus were both affected by the amount of representations to be maintained, retrieved, or processed during the task, independently of their nature.

The BOLD responses in the predefined cingulate and caudate regions were modeled as reflecting two computationally distinct aspects of control: the number of control states required and the amount of information transferred during representational manipulations. This interpretation is crucial to our view of control, and was successful in reproducing the hemodynamic patterns and predicting the dissociation between these structures and the prefrontal regions. A number of alternative accounts, however, must be taken into consideration.

First, trial difficulty correlates with mental effort, which some authors consider the driving force (e.g., Critchley et al., 2003). Our results alone cannot rule out this explanation. Nonetheless, other studies indicate that dACC responses do not decrease with practice, which instead

Table 4. Model's Parameters and Fit

\begin{tabular}{|c|c|c|c|c|c|}
\hline \multirow[b]{2}{*}{ Module } & \multicolumn{3}{|c|}{ Parameter Values } & \multicolumn{2}{|c|}{ Fit } \\
\hline & $a$ (Equation 3) & $s$ (Equation 4) & $M$ (Equation 4) & $r$ & RMSE \\
\hline Manual & 4.0 & 1.0 & 0.14 & .968 & .062 \\
\hline Retrieval & 3.7 & 1.5 & 0.13 & .932 & .058 \\
\hline Goal & 3.7 & 1.1 & 0.23 & .982 & .045 \\
\hline Procedural & 3.9 & 0.9 & 0.05 & .939 & .050 \\
\hline Imaginal & 2.3 & 1.6 & 1.09 & .972 & .066 \\
\hline
\end{tabular}

The three parameters that were estimated independently for each module to simulate the hemodynamic responses in the corresponding regions (see main text for details), together with the corresponding measures of fit with the experimental data. 
reduces difficulty and assuages mental effort (Fincham \& Anderson, 2006).

Second, one could argue that, on a conflict-monitoring perspective, the more difficult conditions have more opportunities for conflict among possible operations, in turn explaining the dACC responses within a conflictmonitoring framework. On the other hand, conflict detection should be followed by some compensative topdown control bias from PFC. However, we failed to find a prefrontal region showing a difference between the internal parametric and external parametric conditions to match the difference in ACC.

Third, activity in the dACC has also been proposed to reflect cognitive preparation, as distinct from the online processing occurring the prefrontal regions (Cole \& Schneider, 2007). More complex equations certainly require more initial preparation. Nonetheless, BOLD responses in ACC seem to extend throughout all the time needed to solve the equation, and not to be limited at an early stage where its difficulty is detected. It is conceivable to stretch this view and envision preparation at a finer-grained scale, essentially occurring before a single cognitive operation. At this level, however, it becomes computationally undistinguishable from the control-state view outlined in this article.

None of these explanations makes explicit predictions about the caudate nucleus, whose activity was also affected by condition difficulty. Brown and Braver (2005), however, suggested that the dACC might contribute to preparation and control simply by reflecting learned taskspecific error likelihoods, which can be used by other regions to adjust for control. Encoding of the error likelihood depends on error-related signals conveyed through the dopamine pathways originating in the basal ganglia, opening the possibility for a similar pattern in both regions. Because, in our experiment, the condition difficulty is correlated with error rates, this alternative explanation cannot be discarded on the basis of our results. However, there are subtle differences in the pattern of responses found in ACC and the caudate nucleus, which emerge naturally in our modeling approach but are not explicitly predicted by this account.

The pattern of activation in both our predefined and exploratory parietal regions was consistently similar to that found in the dACC and the caudate nucleus. Eventually, the hemodynamic responses in the parietal cortices were modeled as reflecting the costs of maintaining and updating intermediate representations of an equation. Our experimental task, however, does not allow for a tight control for these kinds of demands, and our mapping relied on previous experiments (e.g., Anderson, 2005; Anderson et al., 2005). Alternatively, one can imagine parietal regions as playing a role in cognitive control. Furthermore, parietal responses might have been affected by other factors that were not controlled for (such as visual scanning strategies), or were concurrently manipulated in our design (such as memory retrievals).
A number of researchers (e.g., Dehaene, 1997) have suggested that human mathematical problem solving is grounded on specialized brain circuits supporting numerical representations. In fact, activity related to automatic number processing has been found to occur during arithmetic tasks (Dehaene et al., 2003) and even in response to subliminally primed stimuli (Naccache \& Dehaene, 2001). When comparing the two externally updated conditions, however, we could not find any reliable effect due to the mere presence of numbers versus parameters. We cannot rule out the presence of hemodynamic activity reflecting number processing, its effects must have been small compared with the larger ones due to the differences in representational demands. This suggests that, although algebraic problem solving might also be rooted in numerical abilities, it was made possible by those domain-general adaptations that achieved greater control over internal representations and underlie all complex mental activities.

\section{Acknowledgments}

This research was supported by grant FA8750-05-2-0014 from the Defense Advanced Research Projects Agency (DARPA) to John R. Anderson. We thank Jennifer L. Ferris for her comments on previous versions of this manuscript, and Jon $\mathrm{M}$. Fincham for his help through all the stages of this research.

Reprint requests should be sent to Andrea Stocco, Department of Psychology, Carnegie Mellon University, Pittsburgh, PA 15213, orvia e-mail:stocco@cmu.edu.

\section{Notes}

1. A complete list of the equations is available as part of the supplementary model file at: http://act-r.psy.cmu.edu/ publications/pubinfo.php?id $=716$.

2. A comprehensive practice of 24 equations might seem too short. However, an analysis of performance of early (1-3) versus late blocks (5-8) found an effect of blocks on latencies but no effect on accuracies, and no reliable change in the hemodynamic activity was detected.

3. The structural reference image is available at: http://act-r. psy.cmu.edu/mri/.

4. The model is available at: http://act-r.psy.cmu.edu/publications/ pubinfo.php?id $=716$.

5. The strategies for the two internal conditions are essentially similar, both requiring an initial encoding of the right-hand side of the equation, and a subsequent comparison with the addend on the left hand side. The strategy implemented only works for the restricted set of equation used in this experiment.

\section{REFERENCES}

Altmann, E. M., \& Gray, W. D. (2002). Forgetting to remember: The functional relationship of decay and interference. Psychological Science, 13, 27-33.

Anderson, J. R. (2005). Human symbol manipulation within an integrated cognitive architecture. Cognitive Science, 29, 313-341.

Anderson, J. R. (2007). How can the human mind occur in the physical universe? New York: Oxford University Press. 
Anderson, J. R., Albert, M. V., \& Fincham, J. M. (2005). Tracing problem solving in real time: fMRI analysis of the subject-paced Tower of Hanoi. Journal of Cognitive Neuroscience, 17, 1261-1274.

Anderson, J. R., Bothell, D., Byrne, M. D., Douglass, S., Lebiere, C., \& Qin, Y. (2004). An integrated theory of the mind. Psychological Review, 111, 1036-1060.

Anderson, J. R., Qin, Y., Sohn, M.-H., Stenger, V. A., \& Carter, C. S. (2003). An information-processing model of the BOLD response in symbol manipulation tasks. Psychonomic Bulletin E Review, 10, 241-261.

Badre, D., \& Wagner, A. D. (2004). Selection, integration, and conflict monitoring: Assessing the nature and generality of prefrontal cognitive control mechanisms. Neuron, 41, 473-487.

Baird, A., Dewar, B.-K., Critchley, H., Gilbert, S. J., Dolan, R. J., \& Cipolotti, L. (2006). Cognitive functioning after medial frontal lobe damage including the anterior cingulate cortex: A preliminary investigation. Brain and Cognition, 60, 166-175.

Barch, D. M., Braver, T. S., Nystrom, L. E., Forman, S. D., Noll, D. C., \& Cohen, J. D. (1997). Dissociating working memory from task difficulty in human prefrontal cortex. Neuropsychologia, 35, 1373-1380.

Botvinick, M. M., Cohen, J. D., \& Carter, C. S. (2004). Conflict monitoring and anterior cingulate cortex: An update. Trends in Cognitive Sciences, 8, 539-546.

Botvinick, M. M., Nystrom, L. E., Fissell, K., Carter, C. S., \& Cohen, J. D. (1999). Conflict monitoring versus selection-for-action in anterior cingulate cortex. Nature, 402, 179-181.

Botvinick, M. M., \& Plaut, D. C. (2004). Doing without schema hierarchies: A recurrent connectionist approach to routine sequential action and its pathologies.

Psychological Review, 111, 395-429.

Braver, T. S., Cohen, J. D., \& Barch, D. M. (2002). The role of prefrontal cortex in normal and disordered cognitive control: A cognitive neuroscience perspective. In D. T. Stuss \& R. T. Knight (Eds.), Principles of frontal lobe function (pp. 428-447). New York: Oxford University Press.

Braver, T. S., Cohen, J. D., Nystrom, L. E., Jonides, J., Smith, E., \& Noll, D. C. (1997). A parametric study of prefrontal cortex involvement in human working memory. Neuroimage, 5, 49-62.

Brown, J. W., \& Braver, T. S. (2005). Learned predictions of error likelihood in the anterior cingulate cortex. Science, 307, 1118-1121.

Bush, G., Luu, P., \& Posner, M. I. (2000). Cognitive and emotional influences in anterior cingulate cortex. Trends in Cognitive Sciences, 4, 215-222.

Carter, C. S., MacDonald, A. W., III, Botvinick, M. M., Ross, L. L., Stenger, V. A., Noll, D., et al. (2000). Parsing executive processes: Strategic vs. evaluative functions of the anterior cingulate cortex. Proceedings of the National Academy of Sciences, 97, 1944-1948.

Cole, M. W., \& Schneider, W. (2007). The Cognitive Control Network: Integrated cortical regions with dissociable functions. Neuroimage, 37, 343-360.

Critchley, H. D., Mathias, C. J., Josephs, O., O'Doherty, J., Zanini, S., Dewar, B.-K., et al. (2003). Human cingulate cortex and autonomic control: Converging neuroimaging and clinical evidence. Brain, 126, 2139-2152.

Dehaene, S. (1997). The number sense: How the mind creates mathematics. New York: Oxford University Press.

Dehaene, S., Piazza, M., Pinel, P., \& Cohen, L. (2003). Three parietal circuits for number processing. Cognitive Neuropsychology, 20, 487-506.

Dehaene, S., Posner, M. I., \& Tucker, D. M. (1994). Localization of a neural system for error detection and compensation. Psychological Science, 5, 303-305.

Di Pellegrino, G., Ciaramelli, E., \& Làdavas, E. (2007). The regulation of cognitive control following rostral anterior cingulate cortex lesion in humans. Journal of Cognition Neuroscience, 19, 275-286.

Fellows, L. K., \& Farah, M. J. (2005). Is anterior cingulate necessary for cognitive control? Brain, 128, 788-796.

Fincham, J. M., \& Anderson, J. R. (2006). Distinct roles of the anterior cingulate and prefrontal cortex in the acquisition and performance of a cognitive skill. Proceedings of the National Academy of Sciences, 103, 12941-12946.

Fincham, J. M., Carter, C. S., Van Veen, V., Stenger, V. A., \& Anderson, J. R. (2002). Neural mechanisms of planning: A computational analysis using event-related fMRI. Proceedings of the National Academy of Sciences, 99, 3346-3351

Fletcher, P. C., Frith, C. D., Baker, S. C., Shallice, T., Frackowiak, R. S., \& Dolan, R. J. (1995). The mind's eye-Precuneus activation in memory-related imagery. Neuroimage, 2, 195-200.

Forman, S. D., Cohen, J. D., Fitzgerald, M., Eddy, W. F., Mintun, M. A., \& Noll, D. C. (1995). Improved assessment of significant activation in functional magnetic resonance imaging fMRI: Use of a cluster-size threshold. Magnetic Resonance in Medicine, 33, 636-647.

Glover, G. H. (1999). Deconvolution of impulse response in event-related BOLD fMRI. Neuroimage, 9, 416-429.

Graybiel, A. M. (2000). The basal ganglia. Current Biology, 10, R509-R511.

Hill, N. M., \& Schneider, W. (2006). Brain changes in the development of expertise: Neurological evidence on skill-based adaptations. In K. A. Ericsson, N. Charness, P. Feltovich, \& R. Hoffman (Eds.), Cambridge handbook of expertise and expert performance (pp. 653-682). New York: Cambridge University.

Knowlton, B. J., Mangels, J. A., \& Squire, L. R. (1996). A neostriatal habit learning system in humans. Science, 273, 1399-1402.

Koechlin, E., Ody, C., \& Kouneiher, F. (2003). The architecture of cognitive control in the human prefrontal cortex. Science, 302, 1181-1185.

Logan, G. D., \& Bundesen, C. (2003). Clever homunculus: Is there an endogenous act of control in the explicit task-cuing procedure? Journal of Experimental Psychology: Human Perception and Performance, 29, 575-599.

Lovett, M. C. (2005). A strategy-based interpretation of Stroop. Cognitive Science, 29, 493-524.

MacDonald, A. W., III, Cohen, J. D., Stenger, V. A., \& Carter, C. S. (2000). Dissociating the role of the dorsolateral prefrontal and anterior cingulate cortex in cognitive control. Science, 288, 1835-1838.

Miller, E. K., \& Cohen, J. D. (2001). An integrative theory of prefrontal cortex function. Annual Review of Neuroscience, 24, 167-202.

Monsell, S. (2003). Task switching. Trends in Cognitive Sciences, 7, 134-140.

Naccache, L., \& Dehaene, S. (2001). The priming method: Imaging unconscious repetition priming reveals an abstract representation of number in the parietal lobes. Cerebral Cortex, 11, 966-974.

Nolde, S. F., Johnson, M. K., \& D'Esposito, M. (1998). Left prefrontal activation during episodic remembering: An event-related fMRI study. NeuroReport, 9, 3509-3514. 
O'Reilly, R. C., \& Frank, M. J. (2006). Making working memory work: A computational model of learning in the frontal cortex and basal ganglia. Neural Computation, 18, 283-328.

Pardo, J. V., Pardo, P. J., Janer, K. W., \& Raichle, M. E. (1990). The anterior cingulate cortex mediates processing selection in the Stroop attentional conflict paradigm. Proceedings of the National Academy of Sciences, 87, 256-259.

Peebles, D., \& Bothell, D. (2004). Modeling performance in the sustained attention to response task. In C. D. Schunn, M. C. Lovett, C. Lebiere, \& P. Munro (Eds.), Proceedings of the sixth international conference on cognitive modeling (pp. 231-236). Mahwah, NJ: Erlbaum.

Posner, M. I., \& Dehaene, S. (1994). Attentional networks. Trends in Neurosciences, 17, 75-79.

Qin, Y., Carter, C. S., Silk, E., Stenger, V. A., Fissell, K., Goode, A., et al. (2004). The change of the brain activation patterns as children learn algebra equation solving. Proceedings of the National Academy of Sciences, 101, 5686-5691.

Qin, Y., Sohn, M.-H., Anderson, J. R., Stenger, V. A., Fissell, K., Goode, A., et al. (2003). Predicting the practice effects on the blood oxygenation level-dependent (BOLD) function of fMRI in a symbolic manipulation task. Proceedings of the National Academy of Sciences, 100, 4951-4956.

Schneider, D. W., \& Logan, G. D. (2005). Modeling task switching without switching tasks: A short-term priming account of explicitly cued performance. Journal of Experimental Psychology: General, 134, 343-367.

Sohn, M.-H., \& Anderson, J. R. (2001). Task preparation and task repetition: Two-component model of task switching.
Journal of Experimental Psychology: General, 130, 764-778.

Sohn, M.-H., Ursu, S., Anderson, J. R., Stenger, V. A., \& Carter, C. S. (2000). The role of prefrontal cortex and posterior parietal cortex in task-switching. Proceedings of the National Academy of Sciences, 97, 13448-13453.

Swick, D., \& Turken, A. U. (2002). Dissociation between conflict detection and error monitoring in the human anterior cingulate cortex. Proceedings of the National Academy of Sciences, 99, 16354-16359.

Wagner, A. D., Paré-Blagoev, E. J., Clark, J., \& Poldrack, R. A. (2001). Recovering meaning: Left prefrontal cortex guides controlled semantic retrieval. Neuron, 31, 329-338.

Woods, R. P., Cherry, S. R., \& Mazziotta, J. C. (1992). Rapid automated algorithm for aligning and reslicing PET images. Journal of Computer Assisted Tomography, 16, 620-633.

Woods, R. P., Grafton, S. T., Holmes, C. J., Cherry, S. R., \& Mazziotta, J. C. (1998). Automated image registration: I. General methods and intrasubject, intramodality validation. Journal of Computer Assisted Tomography, 22, 139-152.

Yeung, N., Botvinick, M. M., \& Cohen, J. D. (2004). The neural basis of error detection: Conflict monitoring and the error-related negativity. Psychological Review, 111, 931-959.

Yeung, N., Nystrom, L. E., Aronson, J. A., \& Cohen, J. D. (2006). Between-task competition and cognitive control in task switching. Journal of Neuroscience, 26, 1429-1438. 